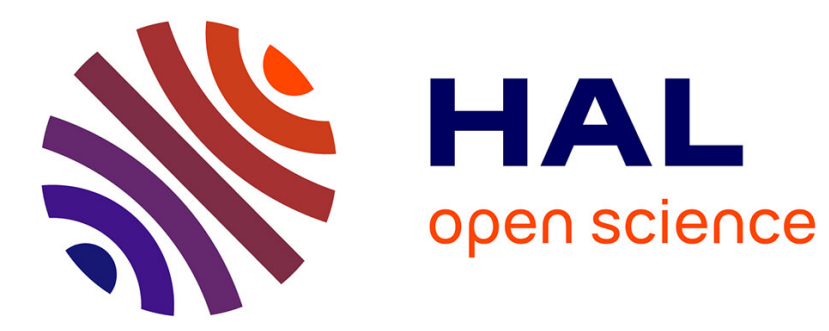

\title{
L'incidence du choix du fait générateur sur la pertinence et la fiabilité des comptes publics : le cas de la Sécurité sociale
}

\author{
Evelyne Lande, Sandrine Boulerne, Fatima Jaouan
}

\section{- To cite this version:}

Evelyne Lande, Sandrine Boulerne, Fatima Jaouan. L'incidence du choix du fait générateur sur la pertinence et la fiabilité des comptes publics: le cas de la Sécurité sociale. Comptabilité - Contrôle Audit, 2008, 14, pp.173-188. 10.3917/cca.142.0173 . halshs-02099258

\section{HAL Id: halshs-02099258 \\ https://shs.hal.science/halshs-02099258}

Submitted on 23 Apr 2019

HAL is a multi-disciplinary open access archive for the deposit and dissemination of scientific research documents, whether they are published or not. The documents may come from teaching and research institutions in France or abroad, or from public or private research centers.
L'archive ouverte pluridisciplinaire HAL, est destinée au dépôt et à la diffusion de documents scientifiques de niveau recherche, publiés ou non, émanant des établissements d'enseignement et de recherche français ou étrangers, des laboratoires publics ou privés. 


\section{L'INCIDENCE DU CHOIX DU FAIT GÉNÉRATEUR SUR LA PERTINENCE ET LA FIABILITÉ DES COMPTES PUBLICS : LE CAS DE LA SÉCURITÉ SOCIALE Évelyne Lande, Sandrine Boulerne et Fatima Jaouan}

Association Francophone de Comptabilité | «Comptabilité - Contrôle - Audit » 2008/2 Tome 14 | pages 173 à 188

ISSN 1262-2788

Article disponible en ligne à l'adresse :

https://www.cairn.info/revue-comptabilite-controle-audit-2008-2-page-173.htm

Distribution électronique Cairn.info pour Association Francophone de Comptabilité.

(C) Association Francophone de Comptabilité. Tous droits réservés pour tous pays.

La reproduction ou représentation de cet article, notamment par photocopie, n'est autorisée que dans les limites des conditions générales d'utilisation du site ou, le cas échéant, des conditions générales de la licence souscrite par votre établissement. Toute autre reproduction ou représentation, en tout ou partie, sous quelque forme et de quelque manière que ce soit, est interdite sauf accord préalable et écrit de l'éditeur, en dehors des cas prévus par la législation en vigueur en France. Il est précisé que son stockage dans une base de données est également interdit. 
L'incidence du choix

du fait générateur sur

la pertinence et la fiabilité

des comptes publics :

le cas de la Sécurité sociale

The impact of the choice

of generating event

on the consolidated accounts

of the social security funds

Évelyne LANDE*, Sandrine BoulERNE** et Fatima JAOUAN***

\section{Résumé}

Cet article a pour objet d'étudier l'impact du choix du fait générateur sur la pertinence de l'information financière, plus particulièrement celle relative aux comptes combinés des organismes de Sécurité sociale. Une analyse théorique et pragmatique des faits générateurs

\section{Abstract}

This paper aims to study the impact of choice of generating event on the relevance of financial information, particularly information on consolidated accounts of the social security funds. A theoretical and pragmatical approach of generating events lead to the adoption of two notions:

* Professeur des Universités, Université de Poitiers

** Maître de conférences, Université de Poitiers, IUT de Poitiers

*** Master Gestion Financière et Fiscale européenne, stagiaire à l'ACOSS 
s'est traduite par l'émergence de notions de fait générateur primaire et fait générateur secondaire dont le choix ne déroge pas aux principes des droits constatés. À l'appui de simulations numériques réalisées sur 3 ans, de 2003 à 2005, sur les comptes combinés des caisses nationales et du régime général, les auteurs démontrent que l'application du fait générateur secondaire d'une part, élimine certains travaux d'inventaire, essentiellement fondés sur des estimations et d'autre part, assure une fiabilité et une pertinence entières des données comptables.

\section{Mots ClÉs. - FAIT GÉNÉRATEUR - PERTinence - Fiabilité - Information COMPTABLE - SÉCURITÉ SOCIALE - COMPTES COMBINÉS}

"primary generating event" and "secondary generating event". The choice between these events doesn't go against the principles of accrual accounting. Numerical simulations realised from 2003 to 2005, on consolidated accounts of social security funds, demonstrate that the "secondary generating event " eliminate period-end accrual, essentially estimated, and provide relevant accounting data.

\section{Correspondance :}

Évelyne LANDE

Université de POITIERS, IAE de POITIERS

Centre de Recherche en Gestion

20, rue Guillaume VII Le Troubadour

BP639

86020 Poitiers CEDEX

Tél. : 0549454479

elande@iae.univ-poitiers.fr

\author{
Sandrine BOULERNE \\ Université de POITIERS, \\ IUT de POITIERS \\ Centre de Recherche en Gestion \\ 20 Rue Guillaume VII Le Troubadour \\ BP639 \\ 86020 Poitiers CEDEX \\ sandrine.boulerne@univ-poitiers.fr
}

\section{Introduction}

La volonté d'améliorer la qualité et la pertinence de l'information comptable et d'unifier la tenue de la comptabilité a conduit les pouvoirs publics à imposer aux différents acteurs de la Sécurité sociale, en 1996, un nouveau référentiel comptable : la comptabilité en droits constatés (appelé aussi comptabilité d'exercice ou d'engagement). L'adoption de ce nouveau référentiel comptable dans les organismes sociaux s'est traduite par l'émergence de la notion de fait générateur des opérations comptables. Le fait générateur d'une opération est l'événement qui provoque, de manière immédiate ou différée, une transaction monétaire ${ }^{1}$;'est donc la date de naissance d'une opération qui se dénouera financièrement ultérieurement. Si la définition d'un fait générateur est relativement bien établie dans le 
cadre d'une activité lucrative, elle apparaît plus complexe pour un organisme de Sécurité sociale où plusieurs dates ou faits générateurs peuvent être valablement retenus.

Or, le choix du fait générateur d'une opération aura de facto des conséquences sur le rattachement des charges et des produits à l'exercice comptable et donc sur le résultat. En effet, en fin d'exercice, les opérations qui ont pris naissance au cours de l'exercice comptable mais qui n'ont pas donné lieu à encaissement ou paiement, sont rattachées à l'exercice comptable sous forme de produits à recevoir (créances), de provisions ou de charges à payer (dettes). Ainsi, le passage aux droits constatés implique de rattacher à un exercice les dépenses et les recettes dès la naissance du fait générateur. Ces opérations d'inventaire sont, par conséquent, différentes selon le fait générateur choisi et cela peut avoir une incidence significative sur l'information financière diffusée.

L'objectif de cet article est d'évaluer et d'analyser l'impact du choix du fait générateur sur l'information comptable et en particulier sur sa fiabilité et sa pertinence pour la prise de décision. En vue de définir le cadre conceptuel de notre étude, les différents faits générateurs envisageables pour la Sécurité sociale, leur impact sur la qualité de l'information diffusée et l'analyse de la pertinence des solutions préconisées seront présentés en première partie. Les différentes pratiques des caisses nationales de la Sécurité sociale seront également recensées et démontreront que la date de comptabilisation des opérations n'est pas uniforme d'une caisse à une autre, introduisant des biais dans l'information diffusée. La seconde partie présente les hypothèses retenues et la méthodologie adoptée. Une simulation numérique sur trois ans sera proposée afin de mesurer l'impact d'un changement de fait générateur sur l'information comptable diffusée, plus précisément sur le montant des produits et charges de la Sécurité sociale et par conséquent sur le résultat qui en découle. Le coût politique induit par un changement de fait générateur sera également mis en évidence.

\section{Lémergence des faits générateurs primaire et secondaire}

\subsection{De l'utilité de l'information comptable au fait générateur}

Parmi les travaux traitant de l'utilité de l'information comptable durant cette dernière décennie, certains se sont focalisés sur les caractéristiques qu'elle doit posséder en vue d'être utilisable. Selon Bruns et McKinnon (1993)², l'information comptable se définit comme étant une donnée chiffrée relative à un phénomène économique passé, présent ou futur d'une entité, ceci à partir d'une observation selon des règles établies. Dés lors, l'information comptable est une information quantitative, générée suivant des normes précises. Même si l'information comptable tend à être restreinte à des flux de trésorerie (comptabilité de caisse) de par cette définition, elle doit également être comprise en termes de flux fondés sur la comptabilité d'engagement afin de déterminer la valeur économique d'une firme, comme le suggère Bierman (1992). Les états financiers ont pour vocation de communiquer l'information comptable. Ils constituent une reddition de comptes et présentent des informations comptables destinées à permettre à des tiers de porter des jugements sur les activités et la gestion financières de la firme. En outre, l'établissement des états financiers réalisés dans le respect de normes proposera une représentation utile de la firme si l'uniformisation dans la présentation de l'information comptable est respectée (Caillau, 1996). 
Pour vraiment avoir un maximum d'utilité pour les décideurs, l'information comptable contenue dans les états financiers doit revêtir deux qualités fondamentales : la pertinence et la fiabilitéb. Si l'un de ces deux aspects qualitatifs de l'information comptable sont remis en cause, ce qui peut être le cas par exemple en présence d'une crise de confiance, alors l'information n'est plus utile aux décideurs, comme le précisent Evraert \& Trebucq (2003) et Haddad \& Khater (2007) :

- pour être pertinente ${ }^{4}$, une information doit permettre d'améliorer la décision en aidant l'utilisateur des états financiers dans son jugement des actions passées, présentes et futures et en confirmant ou en corrigeant ses attentes. Dés lors, l'information doit être une source de réduction de l'incertitude inhérente à une situation, doit être établie et divulguée en temps utile et doit avoir une valeur prédictive et une valeur de confirmation. Selon Evraert (2000), la pertinence est une notion subjective qui change avec l'utilisateur et les décisions à prendre ;

- pour être fiable, une information doit être exempte d'erreurs et de biais significatifs et refléter une image fidèle, neutre et vérifiable de ce qu'elle est censée présenter. Evraert (2000) souligne la nature objective de la fiabilité dés lors qu'elle s’appuie sur la conformité aux normes, règles et procédures.

La pertinence et la fiabilité constituent deux paramètres qui doivent être mis en balance avec le facteur temporel : une information fiable peut ne plus être pertinente si elle est fournie trop tardivement et inversement quelle est l'utilité d'une information pertinente mais se fondant sur des éléments non fiabilisés ? (voir en ce sens, le cadre conceptuel de l'IASC).

Ces deux paramètres n'ont pas toujours été mis en avant lors des différentes études préalables à la mise en place d'une comptabilité en droits constatés au sein des organismes publics (plus particulièrement au sein de la Sécurité sociale), ce qui pose à présent quelques difficultés dans la mise en œuvre opérationnelle des faits générateurs.

La mise en place des droits constatés (ou comptabilité d'engagement) en 1996 a introduit la notion de fait générateur pour la Sécurité sociale : avec une comptabilité de caisse la reconnaissance se faisait au moment du paiement, mais lorsque des droits sont constatés, se pose alors la question de la date de naissance de ces droits et de la date qui sera retenue pour la comptabilisation des opérations. Le choix du fait générateur aura un impact sur le contenu des états financiers ce qui amène à intégrer deux paramètres : le fait générateur retenu permet-il de fournir une information pertinente ? Le fait générateur retenu permet-il de présenter une information fiable ?

Une revue de la littérature sur la notion du "fait générateur » en comptabilité publique ne peut être réalisée dans la mesure où les travaux de recherche sur cette notion sont quasi-inexistants, seules quelques définitions du fait générateur ont été proposées par différents organismes nationaux et internationaux. Les différentes études comparatives au niveau international n'abordent pas cette question très technique et pourtant essentielle quant au contenu de l'information diffusée. Ces études comparatives se focalisent sur les items composants les états financiers (nature des actifs et passifs par exemple) et non sur leur date de reconnaissance. 


\subsection{L'impact du fait générateur sur la fiabilité de l'information comptable}

En 1996, la commission des comptes de la Sécurité sociale (CCSS) diligente un nouveau rapport sur "les méthodes de comptabilisation en droits constatés ${ }^{5}$ ". Le rapport indique que la constitution de provisions pour charges et de produits à recevoir relatifs aux opérations connaissant un dénouement au-delà de la fin de l'année implique l'élaboration de méthodes statistiques:

Suite à ce rapport, les organismes de Sécurité sociale ont été amenés à comptabiliser les opérations comptables en fonction de la date de naissance des droits, ce que nous appellerons le fait générateur primaire. Toutefois, n’aurait-il pas été envisageable de retenir un autre fait générateur, que nous appellerons le fait générateur secondaire, correspondant à la date de déclaration des cotisations ou des prestations reçues?

En effet, le cycle de production de la Sécurité sociale est totalement dépendant de l'activité de ses cotisants et de ses assurés : la Sécurité sociale n'est pas à l'initiative de ses opérations tant en recettes qu'en dépenses, elle est dès lors dépendante de son environnement. La comptabilisation des opérations de la Sécurité sociale peut alors faire référence à la date de naissance du droit (soins, situations ou revenu), ce qui correspond au fait générateur primaire, ou bien à la date d'exigibilité qui correspond à la déclaration des cotisations (demande de prestation ou réception du bordereau récapitulatif de cotisations) ou de liquidation des prestations (traitement des bordereaux et des demandes de prestation), ce qui correspond au fait générateur secondaire. Deux faits générateurs sont alors envisageables :

1. Si la date d'exigibilité est retenue (fait générateur secondaire), on se place à l'intérieur du cycle de production de la Sécurité sociale : les cotisations déclarées d'un exercice servent à payer les remboursements et les prestations demandées ou liquidées dans cet exercice. La Sécurité sociale est en mesure d'appréhender toutes les informations dont elle est destinataire avec certitude puisque les produits seront comptabilisés en fonction des cotisations déclarées et les charges en fonction des demandes de remboursement ou de prestations des assurés reçues et liquidées. Les opérations d'inventaire sont limitées et les données inscrites en comptabilité sont plus fiables dans la mesure où elles se fondent sur des éléments tangibles, les déclarations.

2. Si la date de naissance du droit est retenue (fait générateur primaire), la Sécurité sociale retient un fait générateur symétrique à celui des entreprises qui constatent les dépenses de cotisations par exemple en fonction de la période travaillée et pour les prestations le fait générateur correspondrait à la date de réalisation des soins ou serait fonction de la situation des individus. Néanmoins, les délais de déclaration des cotisations ou de demande de prestations entraînent des écritures d'inventaire lourdes (charges à payer, produits à recevoir, provisions pour risques et charges) et dès lors que la Sécurité sociale souhaite éditer ses comptes rapidement, ces écritures se fondent sur des estimations statistiques des bases de cotisations ou des prestations réalisées. Ces difficultés sont toutefois levées dès l'instant où les enregistrements comptables sont établis en tenant compte des délais de déclaration, et qu'en conséquence les calculs statistiques intègrent les déclarations des cotisants et assurés. Toutefois, la fiabilisation des données statistiques par l'intégration des déclarations aurait pour conséquence que les comptes de la Sécurité sociale ne pourraient être publiés qu’avec des délais incompatibles avec le processus budgétaire et le vote de la loi de Sécurité sociale. 
Ainsi, pour conserver le fait générateur primaire tout en publiant une information fiable, les délais devraient être allongés, ce qui est incompatible avec le processus budgétaire, mais également avec les contraintes d'arrêté des comptes induits par la certification des comptes de la Sécurité sociale (Loi organique $\mathrm{n}^{\circ}$ 2005-881 du 2 août 2005 relative aux lois de financement de la Sécurité sociale). En effet, les opérations d'inventaires (estimation statistique des charges et produits à rattacher à l'exercice) d'un exercice clos au $31 / 12 / \mathrm{N}$, doivent être terminées le 31 janvier $\mathrm{N}+1$ pour que l'ensemble des caisses nationales remontent leurs comptes combinés à l'ACOSS le 8 février $\mathrm{N}+1$. Ce processus laisse un peu plus d'un mois à l'ACOSS pour valider les comptes combinés afin qu'elle les transmette le 15 mars $\mathrm{N}+1$ à la Cour des comptes pour certification (le rapport de la Cour des comptes est remis le 30 juin $\mathrm{N}+1$ ) (figure 1).

\section{Figure 1}

\section{Processus de transmission des informations à l'ACOSS}

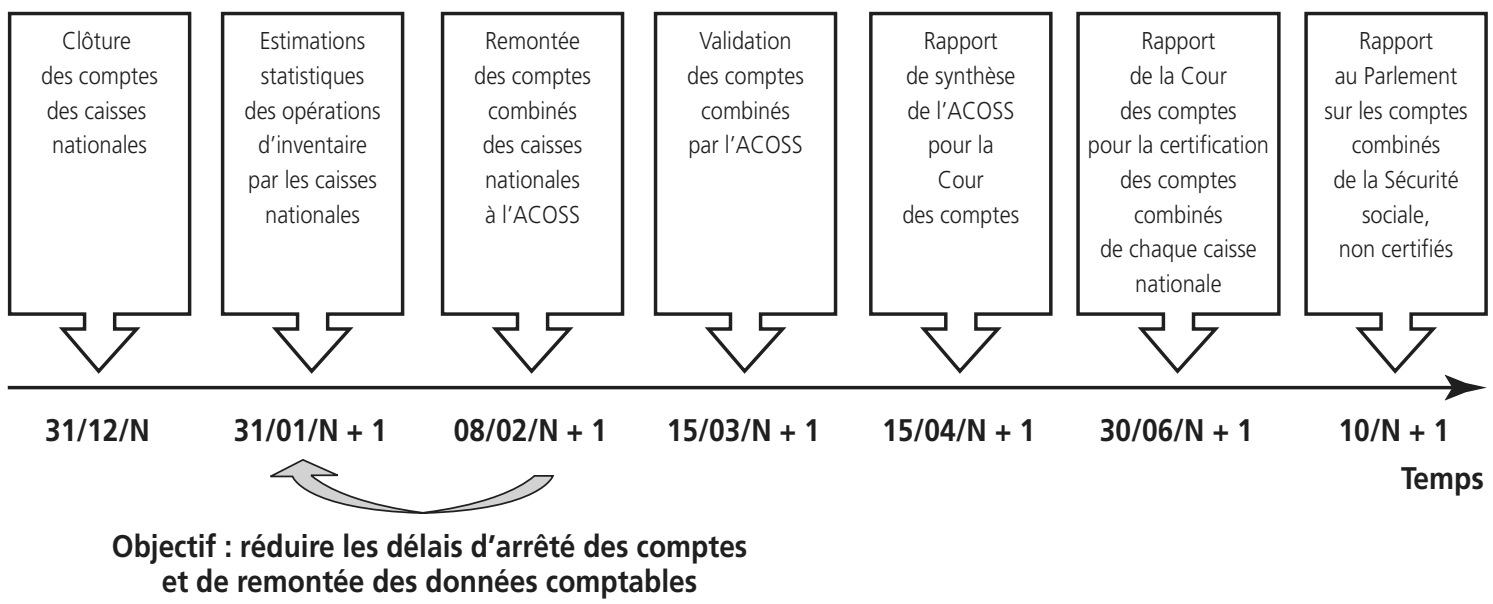

Les contraintes de délai dans l'arrêté des comptes et de remontée de l'information n'induisentelles pas le choix du fait générateur dès lors que l'on souhaite limiter l'incertitude qui y est rattachée ? Ainsi, n’aurait-il pas été plus opportun pour la Sécurité sociale d'opter pour le fait générateur secondaire permettant d'obtenir une information plus fiable en raison des contraintes de délais qu'elle subit et non le fait générateur primaire ? C'est en tout cas ce choix pragmatique qui a été privilégié dans le cadre de l'État français.

L'État français a en effet conditionné le choix du fait générateur à la fiabilité de l'information obtenue. Ainsi, la norme comptable $\mathrm{n}^{\circ} 3$ de l'État relative aux produits régaliens ${ }^{6}$ indique que «Les produits régaliens sont rattachés à l'exercice au cours duquel ils sont acquis à l'État sous réserve qu'ils puissent être mesurés de manière fiable " (recueil des normes comptables de l'État, 2004, ministère de l'Économie, des finances et de l'industrie). Le fait générateur serait ainsi, à titre d'exemple, la réalisation de la matière imposable (impositions). C'est donc le fait générateur primaire qui est à privilégier. Toutefois, dès l'instant où la condition de l'évaluation fiable des produits ne peut pas être respectée, la date d'exigibilité (fait générateur secondaire) est à privilégier si elle permet d'obtenir des données 
comptables plus fiables. Le fait générateur serait alors, par exemple, la déclaration de la matière imposable. Par conséquent, selon le fait générateur choisi, la comptabilisation est réalisée soit à la date de naissance des droits, soit à la date de déclaration de la matière imposable.

Dans quelle mesure, les organismes sociaux ne pourraient-ils pas appliquer le principe de la norme $\mathrm{n}^{\circ} 3$ de l'État, ce qui leur permettrait de limiter les travaux d'inventaire, essentiellement fondés sur des estimations, et aurait un double avantage : permette le raccourcissement des délais de remontée des comptes combinés (remontée au 31 janvier au lieu du 8 février) et de transmettre des informations comptables plus fiables.

Cette question des produits régaliens est également en cours de discussion au sein de l'IPSASB ${ }^{7}$ qui reconnaît, fin 2005, la nécessité d'un complément de réflexion sur la notion de fait générateur pour les organismes sociaux ${ }^{8}$ : le fait générateur survient-il lorsque l'individu répond aux critères d'admissibilité à une prestation ou apparait-il à un stade antérieur ? Cette question reprend notre analyse précédente relative à l'impact du facteur temporel sur le choix du fait générateur.

De même, le projet de norme $e^{9}$ relatif aux "produits des opérations sans contrepartie directe ${ }^{10}$ ", élaboré par l'IPSASB, concerne tout particulièrement les entités composantes de la Sécurité sociale. Ce projet semble adopter les normes conceptuelles américaines selon lesquelles "un revenu sans contrepartie (contribution obligatoire) doit être enregistré lorsque le droit de l'administration existe dans la mesure où la collecte est probable et le montant mesurable ". Cette approche tend vers les normes conceptuelles adoptées par la France et la Nouvelle-Zélande. Si ce projet de norme venait à être validé, les entités adoptant le référentiel de l'IPSASB auraient un délai de 5 ans pour se mettre en conformité.

Il apparaît donc que le débat est loin d'être tranché sur le plan des normes ; le poids des contraintes d'ordre technique (remontée des informations) et temporel (date d'arrêté des comptes) induisant des choix pragmatiques de la part des organismes de Sécurité sociale.

\section{2. Étude de l'impact du fait générateur secondaire sur les comptes combinés de la Sécurité sociale}

\subsection{Les faits générateurs retenus en pratique et l'arbitrage entre exigibilité et naissance du droit}

\subsubsection{LES PRÉCONISATIONS DU PLAN COMPTABLE UNIQUE (PCUOSS)}

Pour la branche maladie, le fait générateur est la liquidation : "réception, instruction et validation $d u$ dossier par le service gestionnaire, des feuilles de soins. Le rattachement est réalisé par rapport à la date des soins avec provisions en fin d'année ». Dans la première partie de cette affirmation, le traitement de la feuille de soins devrait être le fait générateur (soit un fait générateur secondaire) mais dans la seconde partie, le fait générateur serait la date des soins (fait générateur primaire) : un manque de cohérence apparaît dans cette affirmation. Les branches famille et vieillesse ne comptabilisent que les prestations pour lesquelles elles reçoivent une demande, il s'agit bien de la « réception, instruction et vali- 
dation du dossier par le service gestionnaire ". Or tous les dossiers ne sont pas liquidés dans l'exercice (calcul de charges à payer par les CAF et provision pour rappel par la CNAVTS). Le tableau des faits générateurs tiré du Journal officiel peut présenter des différences avec les pratiques des organismes. Ce document est synthétique et général, la branche recouvrement et ses agents ayant à disposition des tableaux beaucoup plus détaillés. Ceci pose le problème de la compréhension par les acteurs de la notion de fait générateur.

\subsubsection{L'ÉVENTUALITÉ DE COMPTABILISER LES COTISATIONS DÉCLARÉES ET LES PRESTATIONS DEMANDÉES}

Le choix du fait générateur implique une méthode de comptabilisation différente des demandes de prestations et des déclarations de cotisations des organismes sociaux. Si le choix du fait générateur se porte sur la naissance du droit des assurés et de la dette des cotisants, des évaluations de produits à recevoir et de provisions sur prestations de la branche maladie doivent être réalisées. Toutefois, sachant que les phénomènes macro-économiques influencent les comportements des agents, ces évaluations risquent de s'écarter amplement de la réalité. Si le fait générateur choisi devenait l'exigibilité, l'estimation de provisions et de produits à recevoir n'aurait plus lieu d'être. Les deux exemples suivants vont permettre d'étayer cette hypothèse :

- pour les prestations des caisses nationales, notamment de la CNAF et de la CNAVTS, le fait générateur est la réception du dossier. Un problème se pose par rapport aux provisions pour rappels, à savoir les évaluations des revalorisations de l'exercice pour la branche famille et les dossiers non liquidés à la clôture de la branche vieillesse. De plus, le système d'information de la branche famille ne permet pas de faire ressortir en $\mathrm{N}+1$ les montants correspondants à des dossiers $\mathrm{N}$ non traités avant le 31 décembre. La fiabilité de la méthode de comptabilisation des charges à payer de la branche famille n'est pas absolue, en raison de contraintes rencontrées lors des comparaisons avec les charges réelles. Ces charges à payer sont simplement contre-passées début $\mathrm{N}+1$. Même constat avec les prestations à régler de la CNAMTS pour lesquelles l'estimation des provisions entraîne toujours une marge d'incertitude. Une comptabilisation, dans les comptes, de montants correspondant aux droits demandés sur un exercice est alors plus fiable. En appliquant le fait générateur « exigibilité », seules les demandes de cet exercice seraient comptabilisées ;

- concernant les contributions des laboratoires et des cotisations des travailleurs indépendants, le fait générateur actuel est la date d'émission des appels de cotisations en raison du caractère très aléatoire des chiffres d'affaires et des revenus. Aucune comptabilisation de produits à recevoir n'est réalisée. Ce choix pragmatique a été arrêté pour plus de fiabilité de l'information dès lors qu'il génère un enregistrement dans les comptes, de données réelles et non évaluées. Les comptes ne peuvent pas inclure les cotisations relatives aux revenus ou chiffres d'affaires de l'exercice, dès l'instant où ils sont déclarés annuellement et au cours de l'exercice suivant. Si ces comptes n'étaient constitués que de provisions, les informations ne feraient preuve d'aucune fiabilité. Comparativement aux cotisations employeurs (données mensuelles), les revenus des travailleurs indépendants et les chiffres d'affaires des laboratoires pharmaceutiques sont déclarés annuellement. Si le même raisonnement est adopté pour les cotisations dues par les employeurs pour lesquels l'estimation porte sur la dernière échéance, la comptabilisation à l'exigibilité permettrait d'avoir dans les comptes uniquement des données réelles et non estimées dont les erreurs faussent le résultat. 
Il semble ici important de rappeler que le choix d'un autre fait générateur ne déroge pas aux principes des droits constatés, la seule différence résulte du décalage entre la naissance du droit (fait générateur primaire) et le moment où il est déclaré et les cotisations calculées (fait générateur secondaire). Ces deux exemples montrent qu'il semble préférable de se fonder sur la date des appels (date d'exigibilité). La question pourra être reposée au moment où les systèmes d'informations et les modes de déclaration seront différents. Si la mission de la Sécurité sociale est de récolter des revenus pour faire face aux besoins des assurés, il est légitime de supposer que les demandes des assurés et les déclarations des cotisants sont deux catégories d'opérations fortement corrélées dans le cycle de production de la Sécurité sociale ${ }^{11}$. En conséquence, si le choix du fait générateur correspond aux demandes des assurés et aux déclarations des cotisants, seules des données fiables et significatives seront comptabilisées dès l'instant où il s'agit des cotisations et contributions devenues exigibles sur cet exercice et de demandes de prestations et de remboursement liquidées sur ce même exercice. Ainsi, les comptes de la Sécurité sociale ne font pas systématiquement preuve d'une fiabilité absolue, au vu des pratiques comptables et des systèmes d'information actuels. Toutes ces considérations amènent la conclusion suivante : la comptabilisation à la date des demandes et déclarations est la méthode la plus fiable pour donner plus de réalité aux comptes de la Sécurité sociale sans pour autant remettre en question la signification du résultat, bien au contraire. En tout état de cause, des améliorations dans les pratiques et les systèmes doivent être étudiées.

À ce stade, il semble important de présenter quels seraient les résultats diffusés par les organismes de Sécurité sociale si au lieu de faire référence au fait générateur primaire il était fait référence au fait générateur secondaire.

\subsection{Méthodologie et simulation numérique}

La problématique développée plus haut conduit à la formulation de deux questions de recherche suivantes :

- un changement dans le choix du fait générateur introduit-il une meilleure pertinence de l'information comptable et génère-t-il une meilleure réactivité ?

- quel est le coût politique induit par un changement de fait générateur ?

Cette étude s'inscrit avant tout dans le cadre général des éléments constitutifs des processus de décisions et pour proposer des éléments de réponses aux interrogations soulevées plus haut, une simulation numérique sur trois ans est réalisée à partir des documents comptables des caisses nationales de la Sécurité sociale :

- la caisse nationale d'assurance maladie - branche maladie (cnam-AM) ;

- la caisse nationale d'assurance maladie - branche accidents du travail (cnam-AT) ;

- la caisse nationale d'assurance vieillesse (cnav) ;

- la caisse nationale d'allocations familiales (cnaf).

Une collaboration de plus de 6 mois avec l'Agence Comptable des Organismes de Sécurité sociale (ACOSS) a été nécessaire pour réaliser cette étude, essentiellement pour l'obtention des estimations statistiques des produits à recevoir et des provisions d'une part et pour une proposition d'une approche méthodologique permettant d'obtenir des données comptables plus pertinentes d'autre part. 
Cet article est écrit avec l'accord de l'ACOSS qui ne souhaite pas que les modalités de calcul des produits à recevoir estimés soient décrites.

Suite à ces estimations statistiques des données d'inventaire, la procédure de calcul située au cœur de l'étude consiste à retraiter les produits et charges techniques (c'est-à-dire les produits et les charges calculés actuellement par référence au fait générateur primaire) des caisses nationales puis du régime général afin d'annuler les opérations d'inventaire (produits à recevoir et provisions), en retranchant les montants estimés comptabilisés à la clôture en $\mathrm{N}$ et en ajoutant ceux contre-passés début $\mathrm{N}+1$.

Ainsi, pour les produits :

PRODUITS FG SECONDAIRE =

PRODUITS FG PRIMAIRE (N) + PRODUITS À RECEVOIR (N-1) - PRODUITS À RECEVOIR (N)

Et pour les charges :

\section{CHARGES FG SECONDAIRE =}

CHARGES FG PRIMAIRE (N) + PROVISIONS (N-1) - PROVISIONS (N)

\section{Le résultat selon le fait générateur secondaire devient alors :}

\section{RÉSULTAT FG SECONDAIRE = RÉSULTAT FG PRIMAIRE (N) + (PRODUITS FG SECONDAIRE \\ - PRODUITS FG PRIMAIRE) - (CHARGES FG SECONDAIRE - CHARGES FG PRIMAIRE)}

Les retraitements et simulations, réalisés sur 3 ans, de 2003 à 2005, sont présentés dans les tableaux 1 et 2 suivants. Ils permettent ainsi de déterminer le résultat de la Sécurité sociale si le fait générateur est l'exigibilité (fait générateur secondaire), et de le comparer au résultat actuel déterminé en fonction du fait générateur primaire. Ces deux résultats seront, à leur tour, comparés à un troisième résultat corrigé des erreurs d'estimation des produits à recevoir et des provisions. En effet, le résultat publié par les organismes de Sécurité sociale est corrigé des sur ou sous estimations des produits à recevoir et des provisions à l'aide du rapport de la CCSS de l'année suivante. Ce résultat corrigé est ainsi plus pertinent que le résultat publié. En conséquence, pour mesurer la fiabilité des comptes, ce résultat corrigé sera pris comme repère et sera comparé au résultat publié actuellement et ainsi qu’au résultat qui intégrerait la notion de fait générateur secondaire.

\section{Analyse des résultats}

À la lecture des données comptables obtenues, les résultats FG secondaire de la Sécurité sociale sont tout de même distants des résultats FG primaire. Une tendance à la baisse apparaît pour les résultats FG secondaire de 2003 et 2005 en comparaison avec les résultats officiels et une tendance à la hausse pour 2004. Dès lors, une analyse des données obtenues permet de déduire que les écarts entre les résultats FG primaire et FG secondaire, exprimés en pourcentage du résultat FG primaire, sont significatifs : 10,5 \% en 2005, 6,9\% en 2004 et de 10,8 \% en 2003. Ces données relatives traduisent l'impact majeur du FG secondaire, fondé sur la date d'exigibilité, sur les résultats FG primaire de la Sécurité sociale. Cette différence entre le résultat FG primaire et le résultat FG secondaire réside dans le contenu informationnel du résultat. Dans le résultat FG primaire, les produits et les charges tiennent compte des prévisions induisant une certaine marge d'incertitude. Au contraire, la détermination du résultat FG secondaire selon le critère de l'exigibilité ne comprend que des produits devenus exigibles et des prestations liquidées au cours de l'exercice, la fiabilité et la pertinence des données comptables sont entières. 

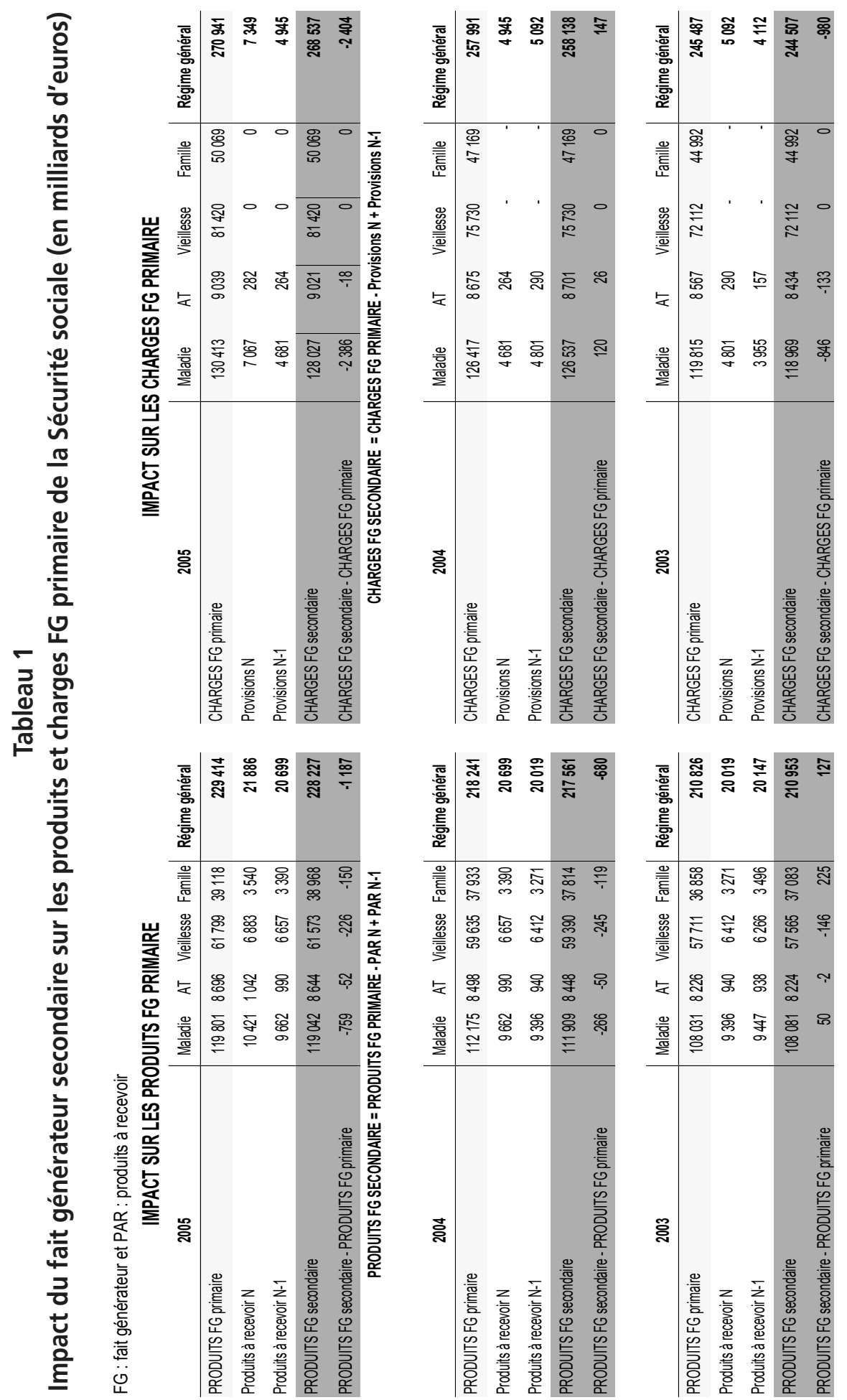

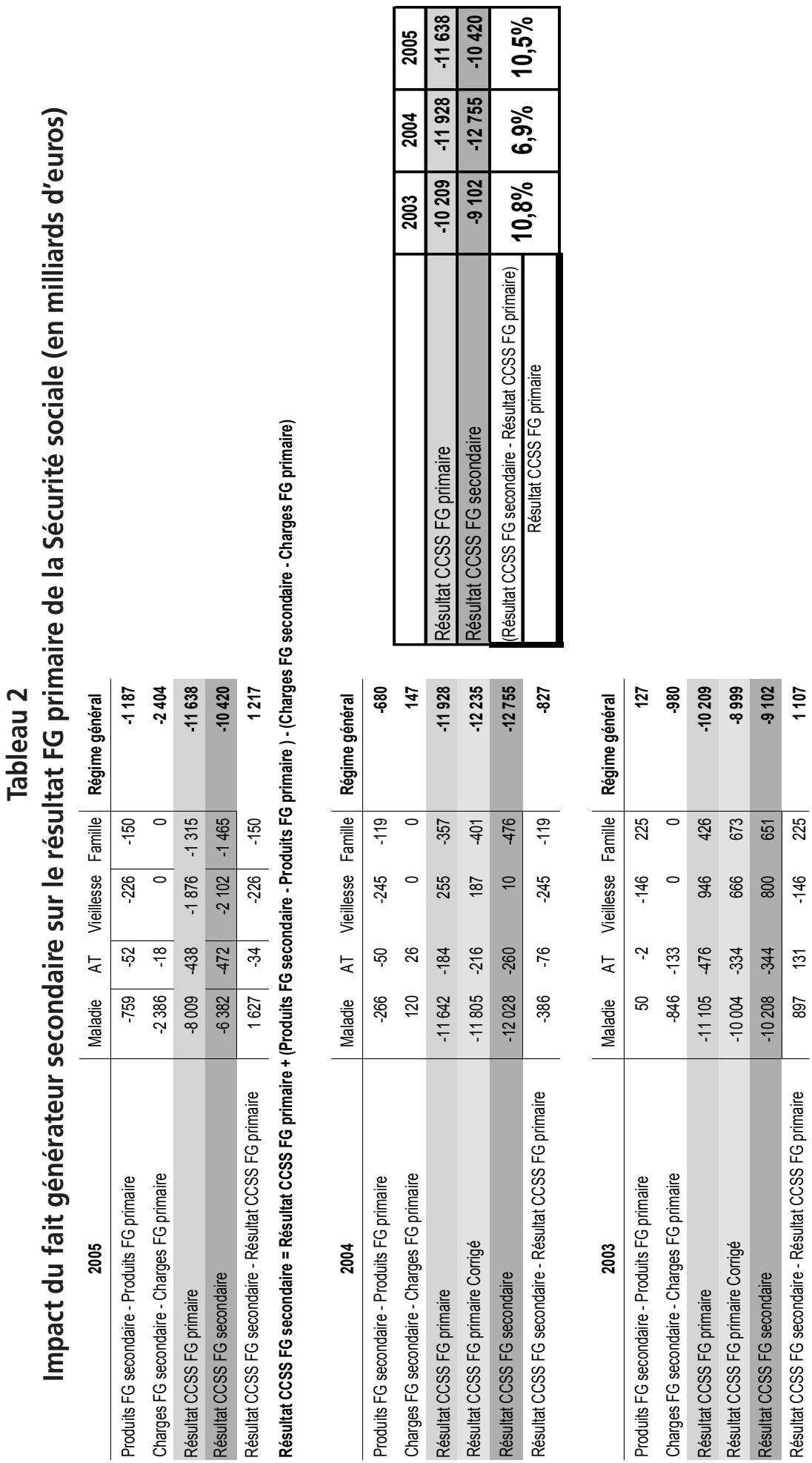
Dés lors, le degré de fiabilité du résultat publié par la Sécurité sociale selon le fait générateur adopté, fait générateur primaire ou fait générateur secondaire, n’est plus le même. Par conséquent, le choix du fait générateur n'est pas sans incidence sur la prise de décision des autorités ministérielles qui pourrait se traduire par un changement de politique sur certaines directives qui avaient été envisagées. La pertinence attribuée au fait générateur secondaire tend à rehausser la qualité de l'information financière et à permettre une meilleure réactivité des autorités concernées lors du processus de décision, ce qui confirme les deux premières hypothèses de notre approche méthodologique.

Toutefois, quel serait l'impact comptable, suite à l'adoption du fait générateur secondaire, sur les produits, les charges et le résultat de la Sécurité sociale?

Une nouvelle simulation est réalisée pour tenter de mesurer cet impact sur les données de 2005.

Tableau 3

Impact sur les comptes de 2005

\begin{tabular}{|l|c|}
\hline \multicolumn{2}{|c|}{ Impact à l'ouverture des comptes sur la situation nette au 1/1/05 } \\
\hline - annulation de produits (produits à recevoir de 2004) & -20699 \\
\hline - annulation de charges (provisions de 2004) & 4945 \\
\hline Impact net & $-\mathbf{1 5 7 5 4}$ \\
\hline \multicolumn{2}{|c|}{} \\
\hline Résultat au 31/12/2005 (fait générateur secondaire) & -10420 \\
\hline \multicolumn{2}{|c|}{} \\
\hline Impact sur la situation nette au 31/12/2005 & $\mathbf{2 6 ~ 1 7 4}$ \\
\hline
\end{tabular}

À la lecture des résultats de cette simulation (tableau 3), l'adoption du fait générateur secondaire, durant l'année de transition 2005, devrait générer une hausse du déficit de la Sécurité sociale de $125 \%$, soit 14536 milliards d'euros ${ }^{12}$. Toutefois, l'application du fait générateur secondaire se traduit par un changement de méthode comptable dès l'instant où elle est justifiée par la recherche d'une meilleure information, condition sine qua non pour qu'un changement de méthode soit considéré comme acceptable. L'impact de ce changement de méthode comptable est à porter en capitaux propres, plus précisément dans le compte "Report à nouveau ", dès l'ouverture de l'exercice. Des informations relatives au contexte doivent être précisées en annexe et les comptes pro forma des exercices antérieurs doivent être produits (art.314-1 du règlement CRC n ${ }^{\circ}$ 99-03)13. Cette procédure comptable n'affecte donc pas le résultat de l'exercice par des corrections d'exercices antérieurs ${ }^{14}$. Selon les données de notre simulation, pour l'exercice 2005, l'impact du changement de méthode évalué à 14536 milliards d'euros, devrait être porté au débit du compte de "Report à nouveau " puisqu'il est négatif. À la clôture de cet exercice, le résultat combiné du régime général ne sera pas affecté par l'impact du changement de méthode. 


\section{Conclusion}

Le passage aux droits constatés crée les conditions d'une image fidèle du patrimoine et de la situation financière de chaque organisme, branche ou régime. Mais les spécificités des opérations de la Sécurité sociale (soins, cotisations, demande de prestations) posent de multiples difficultés au niveau de leur traitement comptable (identification, valorisation et comptabilisation) et expliquent qu'elles restent encore aujourd'hui mal appréhendées par ce nouveau référentiel comptable. En 2006, la Cour des comptes a souligné qu’un effort réel avait été réalisé pour transmettre une information fiable et en phase avec les exigences de la future norme de combinaison, même si un effort important reste à accomplir pour améliorer la qualité et l'exhaustivité du contenu des annexes des comptes combinés. La Cour a également insisté sur une application insuffisante de la comptabilité en droits constatés, tout particulièrement pour les opérations de fin d'exercices, ce qui tendrait à affecter le contenu informationnel et prédictif des données comptables. Pour tenter d'apporter des propositions et des éléments de réflexion à cette dernière critique, les auteurs ont orienté leur étude essentiellement sur l'impact du choix du fait générateur lié à l'exigibilité ou fait générateur secondaire sur la combinaison des comptes de la Sécurité sociale. Les résultats, obtenus suite à des retraitements des données comptables des caisses nationales et à une simulation numérique, traduisent un impact significatif du fait générateur secondaire sur les résultats combinés de la Sécurité sociale durant la période de 2003 à 2005. Pour éviter à la Cour des comptes de constater chaque année des modes de calcul peu explicites des produits à recevoir et des provisions, dans les états financiers des caisses nationales, l'adoption du fait générateur secondaire est la solution la plus sûre. Comme il s'agit d'un changement de méthode comptable, l'incidence sur les comptes sera portée directement en situation nette à l'ouverture et n'affectera pas le résultat dégagé au titre de l'exercice. Enfin, cela permettra à l'ACOSS de raccourcir ses travaux d'inventaire et donc de soumettre plus tôt ses états financiers à la Cour des comptes. 


\section{Notes}

1. À titre d'exemple, dans le cadre de l'assurance maladie, le fait générateur d'une charge technique est la délivrance des soins. En matière de famille ou de retraite, le fait générateur est constitué par la naissance d'un droit.

2. Comme le précisent Bruns et McKinnon, l'association américaine de comptabilité est à l'origine de cette définition.

3. FASB (Financial Accounting Standards Board) : "Qualitative Characteristics of Accounting Information ", Statement of Financial Accounting Concepts n ${ }^{\circ} 2,1980$, p. 21.

4. Cette definition de l'IASB (International Accounting Standards Board) a été discutée en juillet 2006 dans "Preliminary Views on an improved Conceptual Framework for Financial Reporting: The Objective of Financial Reporting and Qualitative Characteristics of Decision-useful Financial Reporting Information ", Discussion paper, July 2006. Cette définition ne diffère pas de celle proposée par le FASB (Financial Accounting Standards Board) en 1980.

5. Rapport final du groupe de travail présidé par Alain Deniel, conseiller maîtreà la Cour des comptes, rapporteur Laurent Gratieux, février 1997

6. Les principes normatifs applicables aux produits régaliens visent à assurer une comptabilisation des produits de l'État conformément aux principes généraux de la comptabilité d'exercice. La nature des produits régaliens perçus sans contrepartie directe pour les tiers constitue en ce sens une spécificité de l'État et de manière générale de l'ensemble des collectivités publiques et n'a pas d'équivalent dans la comptabilité des entreprises. Elle s'illustre principalement par les impôts, taxes assimilées ainsi que les amendes.

7. IPSASB (International Public Sector Accounting Standards Board) : Le Conseil des normes comptables internationales du secteur public, sous la tutelle de la Fédération Internationale des Comptables (IFAC), élabore des normes (IPSAS) selon la méthode de la comptabilité d'exercice qui convergent avec les IFRS en les adaptant au contexte du secteur public.
8. Ou une contrepartie dont la valeur ne correspond pas approximativement à la valeur des biens et services fournis. Pour les mêmes raisons, le champ d'application de l'IPSAS 15 "instruments financiers : information à fournir et présentation" exclut les organismes sociaux. De même pour l'IPSAS 19 "provisions, passifs éventuels et actifs éventuels", qui ne peut, en aucun cas, s'appliquer aux provisions pour prestations sociales fournies par un organisme pour lesquelles celui-ci ne reçoit en retour, directement de la part des bénéficiaires de ces prestations, aucune contrepartie.

9. Un projet de norme, également intitulé « exposésondage ", est un document contenant des propositions relatives à l'application future d'une norme. Ces propositions sont élaborées sur la base des éléments de réponse obtenus suite à des appels à commentaires.

10. Selon l'exposé-sondage 29 (exposure dradft 29 ou ED 29) «Revenue from Non-exchange Transactions (Including Taxes and Transfers", commentaire de Johan Christiaens, Ghent University, 8th February 2006.

11. Excepté la survenue d'un événement exceptionnel.

12. Impact sur la situation nette au 31/12/2005 - Résultat CCSS (fait générateur primaire) $=-26174-(-11638)=-14536$

13. Article 314-1 du règlement $n^{\circ} 99-03$ du Comité de la réglementation comptable. "Lors de changements de méthodes comptables, l'effet après impôt, de la nouvelle méthode est calculé de façon rétrospective, comme si celle-ci avait toujours été appliquée. Dans les cas où l'estimation de l'effet à l'ouverture ne peut être faite de façon objective, en particulier lorsque la nouvelle méthode est caractérisée par la prise en compte d'hypothèses, le calcul de l'effet du changement sera fait de manière prospective. L'impact du changement déterminé à l'ouverture, après effet d'impôt, est imputé en "report à nouveau ", dès l'ouverture de l'exercice sauf si, en raison de l'application de règles fiscales, l'entreprise est amenée à comptabiliser l'impact du changement dans le compte de résultat. Lorsque les changements de méthodes comptables ont conduit à comptabiliser des 
provisions sans passer par le compte de résultat, la reprise de ces provisions s'effectue directement par les capitaux propres pour la partie qui n'a pas trouvé sa justification ".

Cette règle comptable française est en parfaite adéquation avec les dispositions de la norme internationale IAS 8 relative au changement de méthodes comptables.

\section{Références bibliographiques}

Bulletin officiel de la comptabilité publique (2006), Comptabilité de l'État, janvier.

Bierman, Jr.H. (1992), In Defense of Accounting Information, Financial Analyst Journal, 48 (3) : 81-83.

Bruns, Jr.W.J., Mckinnon S.M. (1993), Information and Managers : A Field Study, Journal of Management Accounting Researh, 5 : 84-108.

Caillau J.-C. (1996), Cadre conceptuel de la comptabilité en tant que système de présentation publique de la situation économique (et financière) de l'entreprise, Revue française de comptabilité, 278 : 17-51.

Christiaens J. (2006), Revenue from Non-exchange Transactions Including Taxes and Transfers, comments on the IPSAS Exposure Draft 29, Ghent University.

Conseil national de la comptabilité (2006), Note de présentation afférant au projet d'arrêté relatif aux règles applicables à la combinaison des organismes de la Sécurité sociale - avis n 2006.

Cour des comptes (2006), Rapport préparatoire à la certification des comptes de la Sécurité sociale.

Cour des comptes (2005), Rapport annuel au parlement sur la Sécurité sociale.

Cour des comptes (2005), Rapport de la Cour des comptes de l'État de l'exercice 2005.

Déniel A. (1997), Méthode de comptabilisation en droits constatés; Rapport final du groupe de travail présidé par M. Alain Déniel, Conseiller maître à la Cour des comptes, rapporteur : M. Laurent Gratieux, membre de l'inspection générale des
14. En décembre 2005, la disposition de cet article 314-1 a d'ailleurs été appliquée par la plupart des organismes nationaux en accord avec les composantes dirigeantes de la Sécurité sociale, suite à l'application de changement de méthode comptable résultant d'un contexte différent de celui exposé dans cette étude. Se référer au "Rapport préparatoire à la certification des comptes de la Sécurité sociale ", Cour des comptes, septembre 2006, p. 28-29.

affaires sociales; Commission des comptes de la Sécurité sociale.

Direction de la Sécurité sociale (2005), courrier de l'agence comptable de l'agence centrale des organismes de Sécurité sociale au ministère de la Santé et des solidarités.

Evraert S. (2000), Confiance et comptabilité, in Encyclopédie de Comptabilité, Contrôle de Gestion et Audit, (Eds B. Colasse). Economica, 461-471.

Evraert S. et Trebucq S. (2003), Crise de confiance et information comptable: une étude empirique des réactions du marché français à l'annonce des affaires Enron et Wordcom, 24 ${ }^{\mathrm{e}}$ Congrès de l'AFC, Louvain La Neuve.

Haddad M. et Khater M. (2007), La non fiabilité des informations comptables et les scandales financiers: Cas du groupe mondial "ENRON", Journée internationale de management, Université SaintJoseph de Beyrouth.

Journal officiel (2001), Annexe au $n^{\circ} 291$ Plan comptable unique des organismes de Sécurité sociale.

Ministère de l'Économie, des finances et de l'industrie (2004), Recueil des normes comptables de l'État.

Sécurité sociale (2006), Rapport de la commission des comptes de la Sécurité sociale, Résultats 2005, Prévisions 2006.

Sécurité sociale (2005), Rapport de la commission des comptes de la Sécurité sociale, Résultats 2004, Prévisions 2005 et 2006.

Sécurité sociale (2004), Rapport de la commission des comptes de la Sécurité sociale, Résultats 2003, Prévisions 2004 et 2005. 